\title{
Phenex: Ontological Annotation of Phenotypic Diversity
}

Phenex is a platform-independent desktop application designed to facilitate efficient and consistent annotation of phenotypic variation using Entity-Quality syntax, drawing on terms from community ontologies for anatomical entities, phenotypic qualities, and taxonomic names. Despite the centrality of the phenotype to so much of biology, traditions for communicating information about phenotypes are idiosyncratic to different disciplines. Phenotypes seem to elude standardized descriptions due to the variety of traits that compose them and the difficulty of capturing the complex forms and subtle differences among organisms that we can readily observe. Consequently, phenotypes are refractory to attempts at data integration that would allow computational analyses across studies and study systems. Phenex addresses this problem by allowing scientists to employ standard ontologies and syntax to link computable phenotype annotations to evolutionary character matrices, as well as to link taxa and specimens to ontological identifiers. Ontologies have become a foundational technology for establishing shared semantics, and, more generally, for capturing and computing with biological knowledge.

Phenex can import character-by-taxon matrices in NEXUS format, but, importantly, it uses the new NeXML standard as its native file format. Phenex uses the extensible metadata facilities of NeXML, based on RDFa, to store ontological annotations within character-by-taxon matrix data using explicit semantic relationships. Phenex has been developed in the context of the Phenoscape project, where it has allowed data curators to transform a large body of morphologic character descriptions from the legacy systematics literature into computable phenotype annotations. Homepage: http://phenex.sourceforge.net/ Source available at: http://sourceforge.net/projects/phenex/ License: MIT 